\title{
SPARCL1 suppresses cell migration and invasion in renal cell carcinoma
}

\author{
HUI YE ${ }^{1,2^{*}}$, WEI-GANG WANG ${ }^{3 *}, \mathrm{JUN} \mathrm{CAO}^{1,2}$ and $\mathrm{XI-CHUN} \mathrm{HU}{ }^{1,2}$ \\ ${ }^{1}$ Department of Medical Oncology, Fudan University Shanghai Cancer Center; \\ ${ }^{2}$ Department of Oncology, Shanghai Medical College, Fudan University, Shanghai 200032; \\ ${ }^{3}$ Shanghai Minhang District Gumei Community Health Center, Shanghai 201102, P.R. China
}

Received January 18, 2017; Accepted August 15, 2017

DOI: $10.3892 / \mathrm{mmr} .2017 .7535$

\begin{abstract}
Previous studies have shown that the human SPARC-like 1 (SPARCL1) is crucial for human cancer migration and invasion. In the present study, the expression, biological function and possible molecular regulatory mechanisms of SPARCL1 were investigated in human renal cell carcinoma (RCC). The protein expression of SPARCL1 in cells was evaluated using western blot analysis and immunohistochemical staining in the tissue microarray. The effects of SPARCL1 on the biological behaviors of RCC cells were assessed using in vitro assays. The present study also provisionally investigated the role of SPARCL1 on the mitogen-activated protein kinase (MAPK) signaling pathway. The results revealed that the expression of SPARCL1 was decreased in the RCC cell lines examined and in the tissue microarray. The overexpression of SPARCL1 significantly inhibited cell migration and invasion, and this may have been due to the inactivation of p38/c-Jun N-terminal kinase (JNK)/extracellular signal-regulated kinase (ERK) MAPKs. The results showed that high expression levels of SPARCL1 offered potential as a useful prognostic factor in RCC. Taken together, the present study demonstrated that the expression of SPARCL1 was downregulated in RCC cells and tissues, however, the overexpression of SPARCL1 inhibited RCC cell migration and invasion. SPARCL1 also reduced the expression of phosphorylated p38/JNK/ERK MAPKs. These data suggested that increasing the protein expression level of SPARCL1 may be novel strategy for treating RCC.
\end{abstract}

Correspondence to: Dr Xi-Chun Hu, Department of Oncology, Shanghai Medical College, Fudan University, 130 Dong'an Road, Shanghai 200032, P.R. China

E-mail:huxc16@gmail.com

${ }^{*}$ Contributed equally

Key words: SPARC-like 1, renal cell carcinoma, migration and invasion

\section{Introduction}

Renal cell carcinoma (RCC) accounts for $90 \%$ of renal malignancies and $2-3 \%$ of cases of cancer in adults worldwide $(1,2)$, the incidence of which has been increasing at a rate of $2 \%$ per year. RCC is usually asymptomatic, with a 5-year survival rate of $>90 \%$ for stage I disease and of $20-30 \%$ for stage IV disease $(2,3)$. RCC is resistant to conventional chemotherapy and radiation. The 2004 World Health Organization classified RCC into multiple morphological subtypes: Clear cell RCC, papillary RCC, chromophobe RCC, collecting duct RCC and unclassified forms, which account for 80, 10-15, 5, 1 and $2 \%$ of cases, respectively $(1,4)$.

SPARCL1, also known as MAST9 or hevin, is a member of the SPARC protein family (5). The SPARC family of proteins, includes SPARC, testicans (5), tsc36 (6) and QR1 $(7,8)$. SPARCL1 is downregulated in several types of cancer. In cases of colorectal (9), prostate (10-14), urinary bladder (15), lung (13) and pancreatic cancer (16), SPARCL1 is expressed at low levels. Several studies have demonstrated that SPARCL1 reduces cell proliferation, anchorage-independent growth, migration and invasion in vitro $(5,8,9,13)$ and in vivo $(17,18)$, suggesting that SPARCL1 may function as a tumor suppressor. However, whether SPARCL1 contributes to the progression of $\mathrm{RCC}$ remains to be elucidated.

In the present study, it was found that SPARCL1 was downregulated in human RCC tissues compared with para-carcinoma tissues. When SPARCL1 was overexpressed in RCC cell lines, it was confirmed that SPARCL1 reduced RCC cell migration and invasion, but did not affect growth. Mitogen-activated protein kinase (MAPK) signaling is important in human RCC (19-22). The results of the present study showed that SPARCL1 inhibited the expression of phosphorylated p38/c-Jun N-terminal kinase (JNK)/extracellular signal-regulated kinase (ERK) MAPKs, suggesting that SPARCL1 suppressed RCC cell migration and invasion via the inactivation of $\mathrm{p} 38 / \mathrm{JNK} / \mathrm{ERK} \mathrm{MAPKs}$. Therefore, targeting SPARCL1 may provide an applicable approach for treating RCC.

\section{Materials and methods}

Cells and media. The Caki-1, 768-p, ACTH and HKC cell lines were purchased from the Cell Bank of the Shanghai Institute 
for Biological Science (Shanghai, China). The cells were cultured in Dulbecco's modified Eagle's medium (DMEM), respectively, supplemented with $10 \%$ fetal bovine serum (FBS, Gibco; Thermo Fisher Scientific, Inc., Waltham, MA, USA) at $37^{\circ} \mathrm{C}$ and $5 \% \mathrm{CO}_{2}$.

Tissue microarrays. The RCC tissue microarray, purchased from Wuhan Boster Biological Technology Co., Ltd. (Wuhan, China) was used for the immunohistochemical analysis of the expression of SPARCL1. RCC and para-carcinoma tissue samples have different clinicopathological characteristics including age, gender, tumor volume, Fuhrman classification, pathological tumor stage (pT), pathological lymph node stage $(\mathrm{pN})$, pathological distant metastasis $(\mathrm{pM})$ and pathological tumor-node-metastasis (pTNM) classification.

SPARCL1 vector construction. The HKC human kidney cells were cultured and collected, then RNA was extracted using TRIzol (Invitrogen; Thermo Fisher Scientific, Inc.). Random primers were used to develop the first strand of cDNA. The 5'region of the SPARCL1 gene was isolated using primer pairs cDNA3.1(-)hSPARCL1-NF/cDNA3.1(-) hSPARCL1-SOE-R following denaturation at $98^{\circ} \mathrm{C}$ for $2 \mathrm{~min}, 36$ cycles of $98^{\circ} \mathrm{C}$ for $10 \mathrm{sec}, 52^{\circ} \mathrm{C}$ for $30 \mathrm{sec}, 72^{\circ} \mathrm{C}$ for $90 \mathrm{sec}$, and post-elongation for $10 \mathrm{~min}$ at $72^{\circ} \mathrm{C}$. The mixture comprised 1X PCR buffer, $0.2 \mathrm{mM}$ dNTPs, $0.2 \mu \mathrm{M}$ each primer, 2 units of Primer STAR HS DNA polymerase (cat. no. DR044A; Takara Bio, Inc., Otsu, Japan) and sterile distilled water. Similarly, the intact 3 'region was isolated using cDNA3.1(-)hSPARCL1-SOE-F/hSPARCL1-3'UTR-R and cDNA3.1(-)hSPARCL1-SOE-F/cDNA3.1(-) hSPARCL1-EcoRI-R successively. The full coding region of SPARCL1 was established by splicing overlap extension PCR with the cDNA3.1(-)hSPARCL1-XhoI-F/cDNA3.1(-) hSPARCL1-EcoRI-R primer pairs. Terminally, the SPARCL1 gene was directly cloned into the pcDNA3.1 (-) vector via the XhoI and EcoRI sites. The sequences of the primer pairs are listed in Table I.

SPARCL1 transfection. When the confluence of cells reached $\sim 50-70 \%$, the RCC cells (AHCK, Caki-1 and 769-p), which were cultured in DMEM supplemented with $10 \%$ FCS at $37^{\circ} \mathrm{C}$ in a $5 \% \mathrm{CO}_{2}$ cell culture incubator, were transfected with the pcDNA3.1 (-)SPARCL1 and control vectors using Lipofectamine 2000 (Invitrogen; Thermo Fisher Scientific, Inc., Waltham, MA, USA) according to the manufacturer's protocol. The transfected cells were cultured in DMEM supplemented with $10 \% \mathrm{FBS}$ at $37^{\circ} \mathrm{C}$ in $5 \% \mathrm{CO}_{2}$ for $48 \mathrm{~h}$, and then harvested for analysis. The expression of SPARCL1 was detected using western blot analysis, and in vitro migration and invasion assays were also performed.

Cell viability assays. The cells were seeded in 96-well plates at a concentration of 5,000 cells/well, and cell viability were determined using an MTT assay at 48 or $72 \mathrm{~h}$ according to routine procedures.

Wound-healing assay. Cell migration was assessed using a classic wound-healing assay. The RCC cells were seeded in 6 -well plates at a concentration of $5 \times 10^{5}$ cells/well, and transfected following attachment. Following transfection for 4-6 h, a wound was introduced via a scratch on the monolayer of cells using a $20-\mu 1$ pipette tip, following which the cells were washed with PBS twice to remove non-adherent cells. Images (magnification, x100) of the regions were captured using a photomicroscope at several time points $(0,24,36$ and $48 \mathrm{~h})$.

Transwell invasion assay. For the invasion assay, the upper Transwell chamber was precoated with Matrigel (BD Biosciences, Franklin Lakes, NJ, USA) according to the manufacturer's protocol. At $48 \mathrm{~h}$ post-transfection, the cells were harvested, counted and suspended in serum-free DMEM. Subsequently, $1 \times 10^{5}$ cells in $100 \mu 1$ medium were added to the upper chamber. Medium containing 10\% FBS was added to the lower chamber as a chemoattractant. Following incubation at $37^{\circ} \mathrm{C}$ for $24 \mathrm{~h}$, the cells in the chambers were fixed with $4 \%$ paraformaldehyde for $20 \mathrm{~min}$, following which $0.1 \%$ crystal violet solution was added for $15 \mathrm{~min}$, and then immersed in phosphate-buffered saline (PBS) for $20 \mathrm{~min}$. Finally, the cells in the lower chamber were counted under an inverted microscope. The cell numbers in five randomly selected fields of view were counted (magnification, $\mathrm{x} 100$ ).

Immunohistochemistry. Tissue microarray sections $(5-\mu \mathrm{m})$ of the formalin-fixed and paraffin-embedded specimens were deparaffinized using xylene and rehydrated in graded ethanol. The samples were then preincubated with $3 \% \mathrm{H}_{2} \mathrm{O}_{2}$ to inhibit endogenous peroxide activity. The sections were then incubated at $4^{\circ} \mathrm{C}$ overnight with primary antibody against SPARCL1 (cat. no. 2728; 1:50; R\&D Systems, Inc., Minneapolis, MN, USA) as described previously $(16,23)$, and then with horseradish peroxidase (HRP)-labeled secondary antibodies (cat. no. KIT-5004; $50 \mu \mathrm{l}$; MaxVision, Fuzhou, China) at room temperature for $15 \mathrm{~min}$, followed by the addition of freshly prepared 3,3'-diaminobenzidine for color development for $5 \mathrm{~min}$. In the control group, the primary antibody was replaced with PBS. The immunohistochemical images were obtained with a Leica microscope (Leica Microsystems $\mathrm{GmbH}$, Wetzlar, Germany) and immunohistochemical staining was evaluated independently by two examiners, who were blinded to the clinicopathological information. The intensity of the immunostaining was assessed and assigned scores as follows: 0 , negative staining; 1 , low staining intensity; 2 , moderate staining intensity; 3 , high staining intensity.

Western blot analysis. For western blot analysis, the cells were harvested and washed twice with ice-cold PBS, followed by lysis with ice-cold lysis buffer (4\% sodium dodecyl sulfate, $20 \%$ glycerol and $0.2 \mathrm{M}$ dithiothreitol). Protein concentrations were determined using the BCA method (Beyotime Institute of Biotechnology, Haimen, China). Then the samples were separated by $10 \%$ SDS-PAGE, transferred onto nitrocellulose membranes (EMD Millipore, Billerica, MA, USA), and hybridized $4^{\circ} \mathrm{C}$ overnight with anti-ERK antibody (cat. no. 9102; 1:1,000; Cell Signaling Technology, Inc., Danvers, MA, USA), anti-p38 antibody (cat. no. 9212; 1:1,000; Cell Signaling Technology, Inc.), anti-JNK antibody (cat. no. 9252; 1:1,000; Cell Signaling Technology, Inc.), anti-p-ERK antibody (cat. no. 9101; 1:1,000; Cell Signaling Technology, Inc.), anti-p-p38 antibody (cat. no. 9211; 1:1,000; Cell Signaling Technology, 
Table I. Sequences of primers.

\begin{tabular}{ll}
\hline Name & \multicolumn{1}{c}{ Sequence (5'-3') } \\
\hline cDNA3.1(-)hSPARCL1-NF & CAACTGAAGGTACATTGGACATA \\
cDNA3.1(-)hSPARCL1-XhoI-F & ACGGGCCCTCTAGACTCGAGATGAGTGAGCCTCAGGAGAAA \\
cDNA3.1(-)hSPARCL1-EcoRI-R & TAGTCCAGTGTGGTGGAATTCTCAAAACAAGAGATTTTCATCTATG \\
CDNA3.1(-)hSPARCL1-Soe-F & AATGAAAATATAGGTACCACTGAGC \\
cDNA3.1(-)hSPARCL1-Soe-R & GCTCAGTGGTACCTATATTTTCATT \\
hSPARCL1-3'UTR-R & TACAAGTATCACAGCTGCAT
\end{tabular}

F, forward; R, reverse; SPARCL1, SPARC-like 1.

A

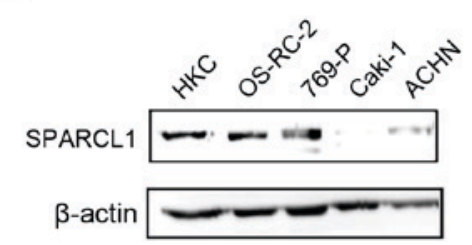

C

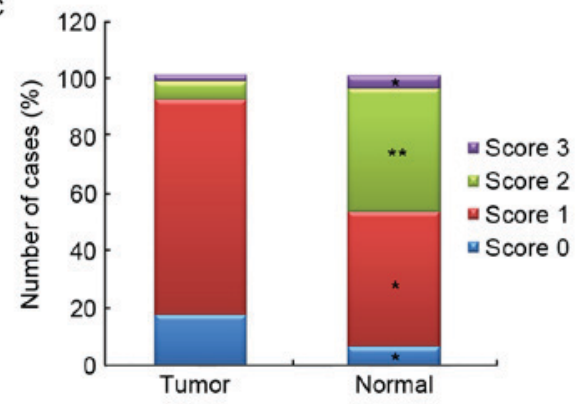

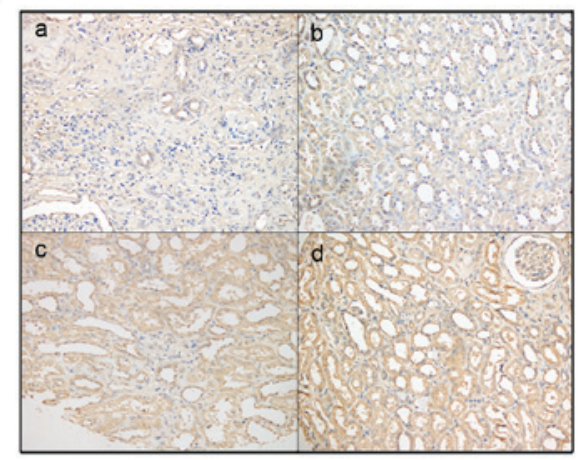

D

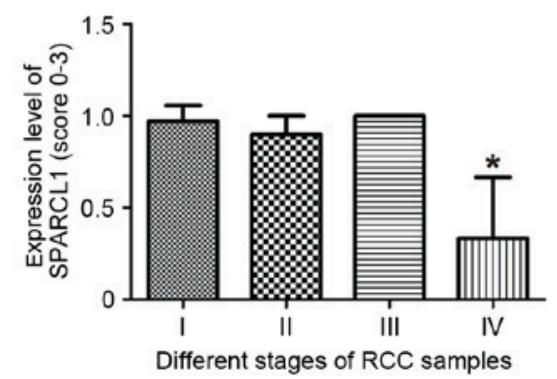

Figure 1. Expression levels of SPARCL1 are downregulated in RCC cells and tissues. (A) Expression of SPARCL1 was detected using western blot analysis in RCC cells. (B) Representative images of immunohistochemical staining of SPARCL1 in RCC tissues and paired non-cancerous tissues: (a) score 0; (b) score 1; (c) score 2; (d) score 3 (magnification, $x 100$ ). (C) Quantification of SPARCL1 staining in the RCC tissue microarray. ${ }^{*} \mathrm{P}<0.05$ and ${ }^{* *} \mathrm{P}<0.01$ in tissues with the same score between RCC and paired non-cancerous tissues. (D) Analysis of the association between the expression of SPARCL1 and pTNM classification. ${ }^{*} \mathrm{P}<0.05$, compared with the stages I-III. RCC, renal cell carcinoma; SPARCL1, SPARC-like 1.

Inc.), anti-p-JNK antibody (cat. no. 4668; 1:1,000; Cell Signaling Technology, Inc.), anti-SPARCL1 antibody and anti-actin antibody (cat. no. ab8226; 1:2,000; Abcam, Cambridge, UK). The immune complexes were detected by incubation with anti-rabbit or anti-mouse IgG antibodies conjugated to HRP (cat. nos. 111-035-003, and 115-035-003; 1:3,000; Jackson ImmunoResearch Laboratories Inc., West Grove, PA, USA) for $1 \mathrm{~h}$ at $37^{\circ} \mathrm{C}$, followed by ECL detection (Thermo Fisher Scientific, Inc.) according to the manufacturer's protocol.

Statistical analysis. Statistical analysis was performed using GraphPad Prism version 5.00 (GraphPad Software, Inc., San Diego, CA, USA). Data are expressed as the mean \pm standard error of the mean. Comparisons between two conditions were analyzed using two-tailed unpaired t-tests when the data were normally distributed; otherwise Mann-Whitney analysis was performed. $\mathrm{P}<0.05$ was considered to indicate a statistically significant difference.

\section{Results}

Expression of SPARCL1 is downregulated in RCC cells. To examine the possible role of SPARCL1 in RCC, western blot analysis was performed to quantify the protein levels of SPARCL1. The results showed that the expression of SPARCL1 was significantly reduced in the RCC cells, compared with that in the normal HKC cells (Fig. 1A).

The expression levels of SPARCL1 were also detected in 50 primary RCC and paired non-cancerous tissues using the tissue microarray and immunohistochemical staining techniques. Images showing the four expression levels of SPARCL1 (0, negative staining; 1 , low staining intensity; 2 , 
A

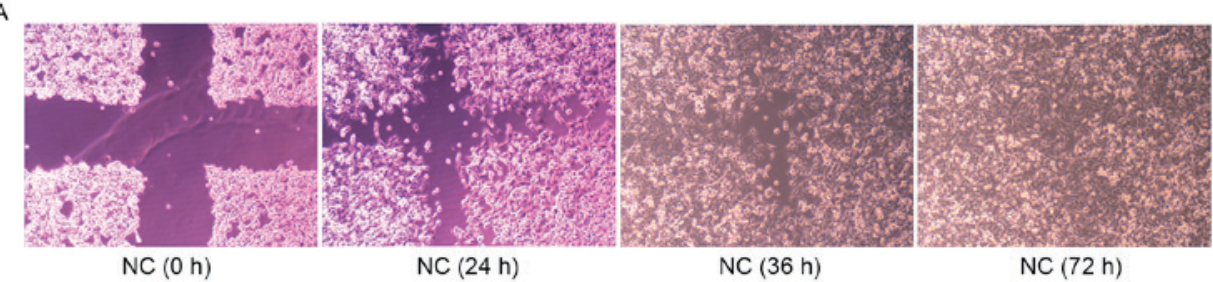

B

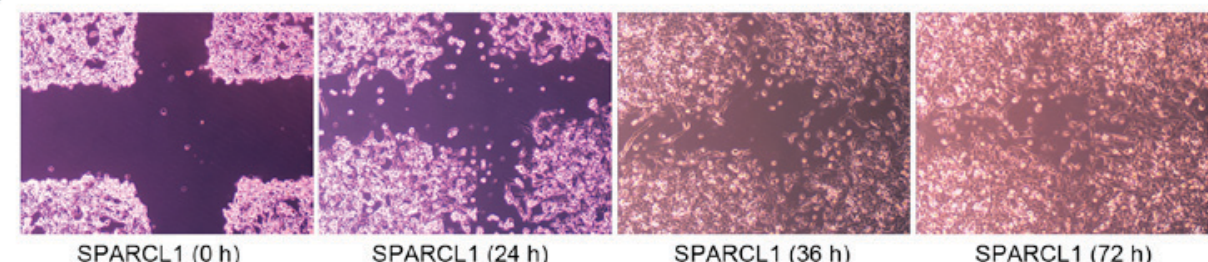

C

D
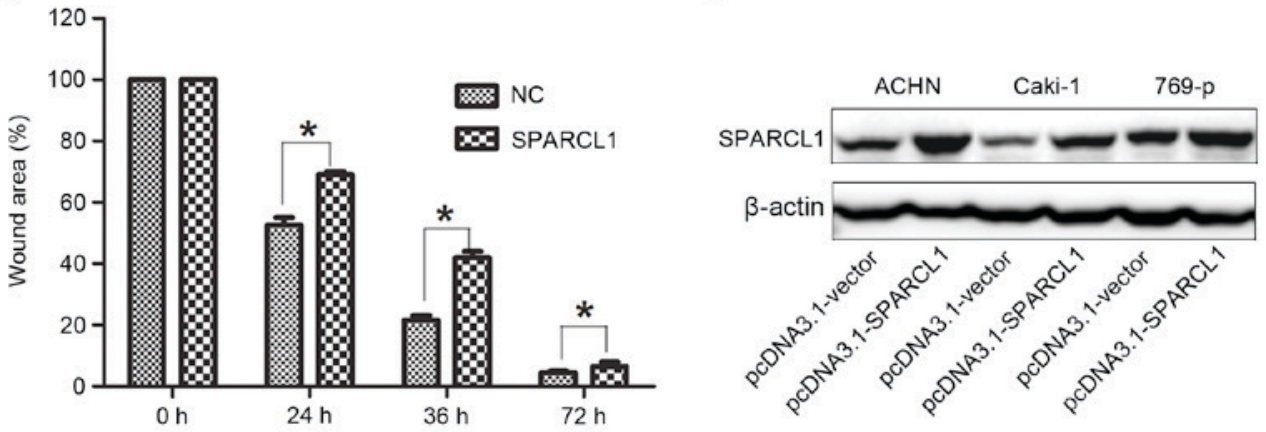

Figure 2. SPARCL1 inhibits the migration of human RCC cells in vitro. Wound healing migration assays were performed using AHCN cells following transfection with (A) NC or (B) SPARCL1. Microscopic observations were recorded at $0,48,36$ and $72 \mathrm{~h}$ following scratching of the cell surface (magnification, $\mathrm{x} 100)$. Representative images from each independent experiment are shown. (C) Quantitative analyses of wound closure rates and cell invasion. The percentage of the wound healing was calculated as: (width of wound at $0 \mathrm{~h}$-width of wound at $72 \mathrm{~h}$ )/width of wound at $0 \mathrm{~h}$. (D) Overexpression of SPARCL1 in RCC cells was detected using western blot analysis. All experiments were performed in triplicate, "P $<0.05$. RCC, renal cell carcinoma; SPARCL1, SPARC-like 1; NC, negative control.

moderate staining intensity; 3 , high staining intensity) are shown in Fig. 1Ba-d. The results showed that the expression levels of SPARCL1 in the RCC tissues differed substantially from those in the normal non-cancerous tissues. In the paired normal tissues, $46.7 \%$ of the tissues showed moderate or high expression of SPARCL1, whereas this was only observed in $8.35 \%$ of the RCC tissues (Fig. 1C). Absent or low expression levels of SPARCL1 were observed in $91.65 \%$ of the tumor samples, however, this was only found in $53 \%$ of the paired normal tissues (Fig. 1C). Among the 50 RCC samples, 41 samples were stage I, five samples were stage II, one sample was stage III and three samples were stage IV. The association between expression and pTNM classification was analyzed, and it was found that the expression of SPARCL1 was significantly reduced in stage IV samples (Fig. 1D). This is a preliminary finding due to the small number of patients in the tissue microarray.

SPARCL1 inhibits migration and invasion, and promotes the proliferation of human RCC cells in vitro. Metastasis is a problem central to cancer therapeutics, therefore, the present study aimed to assess whether the overexpression of SPARCL1 affected tumor migration and invasion. The overexpression of SPARCL1 was induced in RCC cells, following which migration and invasion assays were performed. As shown in Fig. 2, an inverted microscope was used to observe the differences in the wounded regions in the ACHN cell cultures at 24, 36 and $72 \mathrm{~h}$. The overexpression of SPARCL1 markedly reduced the number of migrated cells. As shown in Fig. 2A, the region damaged by the scratch in the control group was infiltrated with migrated RCC cells. In the cells overexpressing SPARCL1, fewer cells were found to have migrated into the scratch area (Fig. 2B). The numbers of cells were counted in the wounded regions of the groups at different time points (Fig. 2C). The overexpression of SPARCL1 in the RCC cells was confirmed using western blot analysis prior to migration and invasion assays (Fig. 2D). These results suggested that SPARCL1 had an inhibitory effect on RCC cell migration. The inhibitory effect of SPARCL1 on migration was also confirmed in the Caki-1 RCC cell line (Fig. 3A-C).

As shown in Fig. 4A and B, the overexpression of SPARCL1 markedly reduced the number of cells migrating to the lower side of the membrane in the Caki-1 cells. Counting of the invasive cells per field was performed, as shown in Fig. 4C. The effect of SPARCL1 on the proliferation of RCC cell lines was detected using MTT assays (Fig. 5A-C). The results showed that the proliferation rates of the Caki-1, ACHN and 769-p cells were accelerated by the overexpression of SPARCL1 for 
A

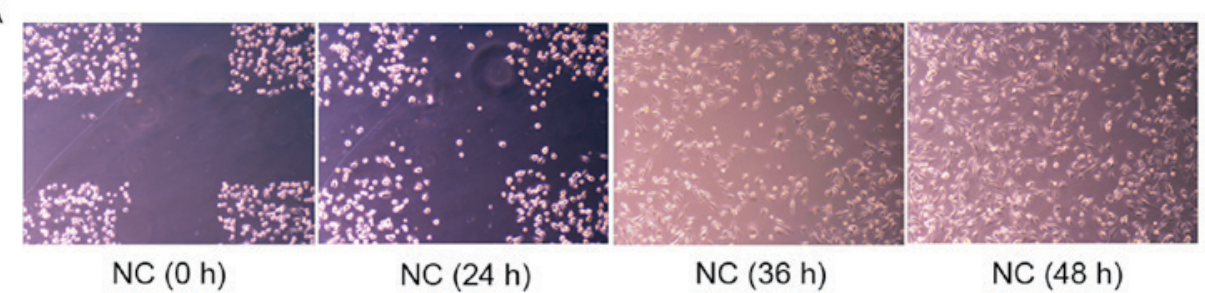

B
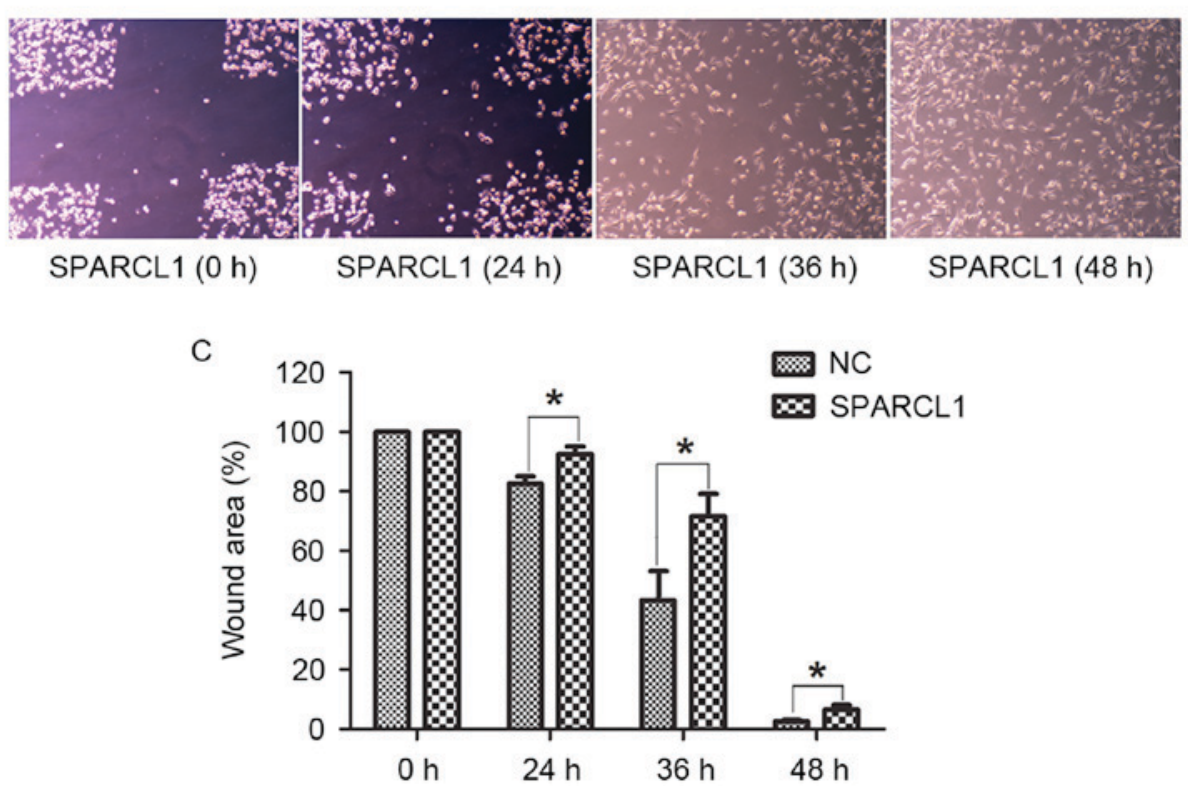

Figure 3. SPARCL1 inhibits the migration of human renal cell carcinoma cells in vitro. Wound healing migration assays were performed in Caki-1 cells following transfection with (A) NC or (B) SPARCL1. Microscopic observations were recorded at 0,24,36 and $48 \mathrm{~h}$ following scratching of the cell surface (magnification, x100). Representative images from each independent experiment are shown. (C) Quantitative analyses of the wound closure rates and cell invasion. The percentage of the wound healing was calculated as: (width of wound at $0 \mathrm{~h}$-width of wound at $48 \mathrm{~h}$ )/width of wound at $0 \mathrm{~h}$. All experiments were performed in triplicate, ${ }^{*} \mathrm{P}<0.05$. SPARCL1, SPARC-like 1 ; NC, negative control.

A

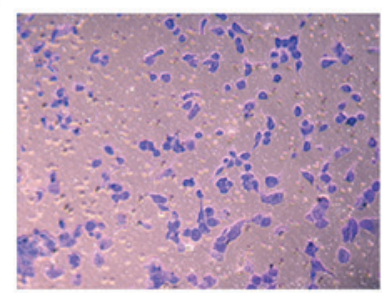

NC $(48 \mathrm{~h})$

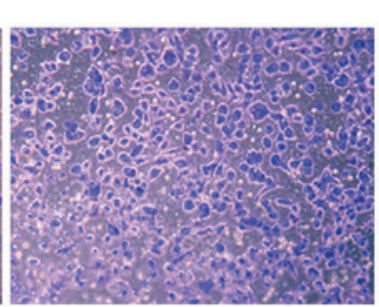

NC $(72 \mathrm{~h})$

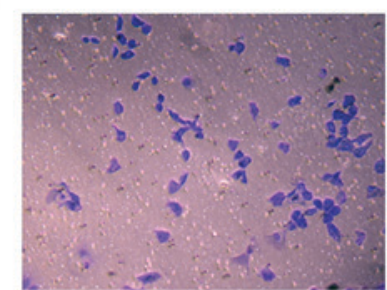

SPARCL1 (48 h)

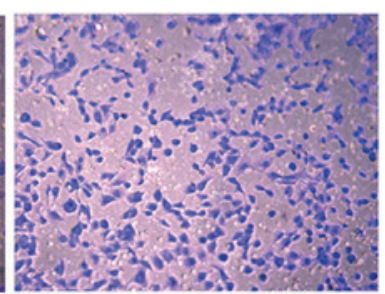

SPARCL1 (72 h)

C

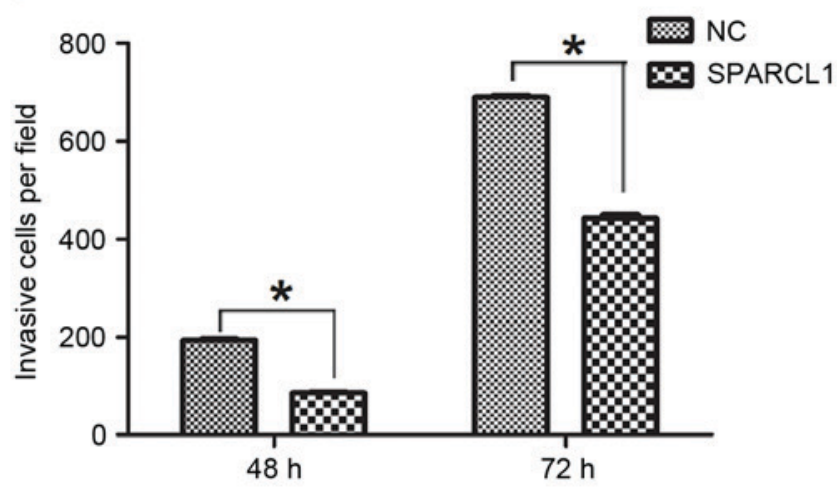

Figure 4. SPARCL1 inhibits the invasion of human renal cell carcinoma cells in vitro. Transwell invasion assays with Matrigel were performed in Caki-1 cells following transfection with (A) NC or (B) SPARCL1 at 48 and $72 \mathrm{~h}$ (magnification, x100). (C) Quantitative analysis of cell invasion. "P<0.05. SPARCL1, SPARC-like 1; NC, negative control. 
A

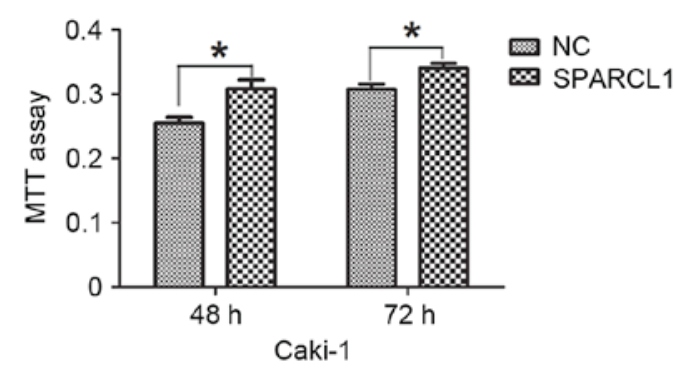

C

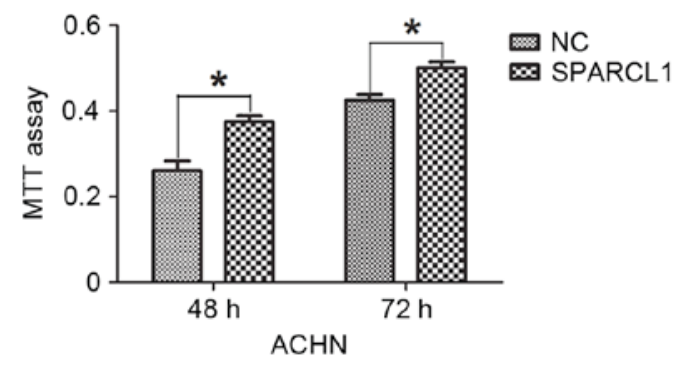

B

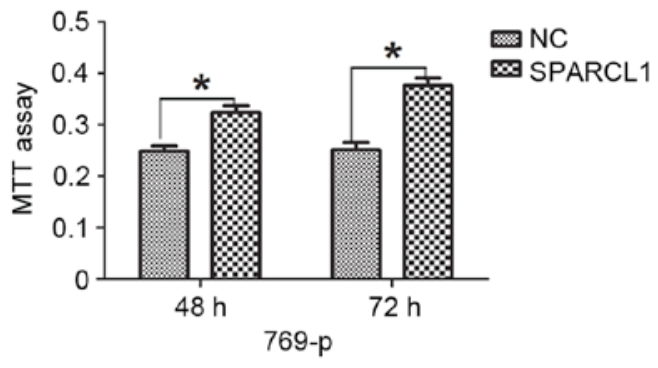

D

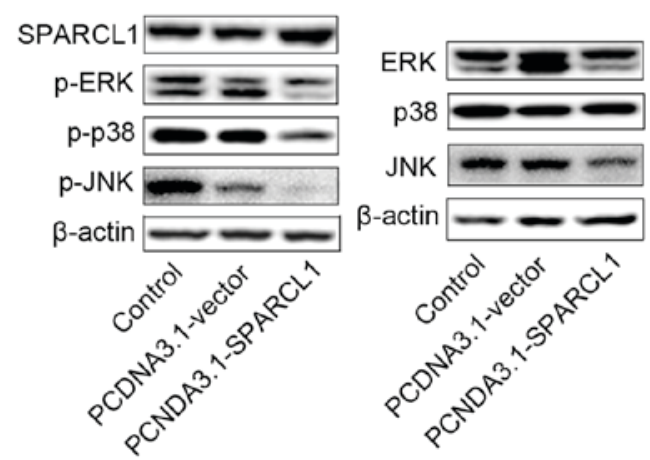

Figure 5. SPARCL1 promotes the proliferation of RCC cells and reduces the activation of p38/JNK/ERK mitogen-activated protein kinases. (A-C) RCC cells were transfected with NC or SPARCL. After 48 and $72 \mathrm{~h}$, cell numbers were assessed using an MTT assay. Statistical analysis with Student's t-test showed a significant increase in cell growth when SPARCL1 was overexpressed. "P<0.05. (D) Western blot analysis of the protein expression levels of total p38, JNK and ERK, and p-p38, p-JNK and p-ERK in RCC cells. RCC, renal cell carcinoma; SPARCL1, SPARC-like 1; NC, negative control; JNK, c-Jun N-terminal kinase; ERK, extracellular signal-regulated kinase; p-, phosphorylated.

48 or $72 \mathrm{~h}$, respectively, suggesting that SPARCL1 promoted RCC cell proliferation.

SPARCL1 inhibits activation of the p38/JNK/ERK MAPK pathway. MAPK kinases are crucial enzymes at the intersection of several biological pathways, which regulate cell differentiation, proliferation and survival. MAPK comprises three protein kinases: JNK, p38 and ERK. In vitro, the overexpression of SPARCL1 appeared to significantly reduce the phosphorylation of JNK, ERK and p38 proteins in the RCC cells (Fig. 5D). These results showed that SPARCL1 may contribute to inactivation of the JNK/ERK/p38 signaling pathway, and lead to the inhibition of RCC cell proliferation and migration.

\section{Discussion}

The present study analyzed the results of SPARCL1 immunohistochemical staining, and found that almost $92 \%$ of the RCC samples were SPARCL1-negative or low intensity, and only $8 \%$ of the RCC samples were SPARCL1-moderate or high intensity. It was found that $\sim 54 \%$ of the normal tissues were SPARCL1-negative or showed low intensity positive staining, and almost $46 \%$ of the normal tissues were SPARCL1-positive at a moderate or high intensity. These results showed that the expression of SPARCL1 was significantly reduced in RCC tissues, comparison with that in normal tissues. It was also found that the expression of SPARCL1 was significantly reduced at stage IV. This suggested that a high expression of
SPARCL1 offers potential as a prognostic factor. Future investigations aim to increase the sample size to investigate the association between the expression of SPARCL1 and kidney staging.

SPARCL1 is downregulated in several types of cancer, which inhibits cancer cell growth, migration or invasion, suggesting that SPARCL1 may function as a tumor suppressor $(2,11-13,16)$. The present study found that the expression levels of SPARCL1 were significantly reduced in RCC cells. Using traditional in vitro approaches to examine the function of SPARCL1, the present study on RCC cell lines demonstrated that SPARCL1 decreased the migratory and invasive properties of RCC cells but did not restrict the growth of the RCC cells. SPARCL1 may be a clinically useful biomarker with diagnostic, prognostic and therapeutic value.

MAPK pathways are crucial in regulating cell differentiation (24-26), proliferation (27-30) and survival (31-33). Mutations of the MAPK pathway proteins have been reported to be associated with several types of human cancer (34-38). MAPK signaling has been shown to be crucial in tumor genesis and tumor metastasis (39-41). The overexpression of MAPK was also found in 52\% of human RCC cases in a previous study (42). In the present study, it was found that SPARCL1 downregulated the expression levels of p-p38, p-JNK and p-ERK in the RCC cells, suggesting that SPARCL1 may regulate the progression of RCC through the p38/JNK/ERK MAPK pathway. Further investigations are required to examine the effect and mechanism of SPARCL1 in treating RCC. 


\section{References}

1. Rini BI, Campbell SC and Escudier B: Renal cell carcinoma Lancet 373: 1119-1132, 2009.

2. Chow WH, Dong LM and Devesa SS: Epidemiology and risk factors for kidney cancer. Nat Rev Urol 7: 245-257, 2010

3. Lopez-Beltran A, Carrasco JC, Cheng L, Scarpelli M, Kirkali Z and Montironi R: 2009 update on the classification of renal epithelial tumors in adults. Int J Urol 16: 432-443, 2009.

4. Lopez-Beltran A, Scarpelli M, Montironi R and Kirkali Z: 2004 WHO classification of the renal tumors of the adults. Eur Urol 49: 798-805, 2006.

5. Brekken RA and Sage EH: SPARC, a matricellular protein: At the crossroads of cell-matrix communication. Matrix Biol 19: 816-827, 2001

6. Vannahme C, Smyth N, Miosge N, Gösling S, Frie C, Paulsson M, Maurer P and Hartmann U: Characterization of SMOC-1, a nove modular calcium-binding protein in basement membranes. J Bio Chem 277: 37977-37986, 2002.

7. Vannahme C, Gösling S, Paulsson M, Maurer P and Hartmann U: Characterization of SMOC-2, a modular extracellular calcium-binding protein. Biochem J 373: 805-814, 2003.

8. Yan Q and Sage EH: SPARC, a matricellular glycoprotein with important biological functions. J Histochem Cytochem 47: 1495-1506, 1999

9. Yu SJ, Yu JK, Ge WT, Hu HG, Yuan Y and Zheng S: SPARCL1, Shp2, MSH2, E-cadherin, p53, ADCY-2 and MAPK are prognosis-related in colorectal cancer. World J Gastroenterol 17: 2028-2036, 2011.

10. Taylor BS, Schultz N, Hieronymus H, et al: Integrative genomic profiling of human prostate cancer. Cancer Cell 18: 11-22, 2010.

11. Chandran UR, Ma C, Dhir R, Bisceglia M, Lyons-Weiler M, Liang W, Michalopoulos G, Becich M and Monzon FA: Gene expression profiles of prostate cancer reveal involvement of multiple molecular pathways in the metastatic process. BMC Cancer 7: 64, 2007.

12. Hurley PJ, Marchionni L, Simons BW, Ross AE, Peskoe SB, Miller RM, Erho N, Vergara IA, Ghadessi M, Huang Z, et al: Secreted protein, acidic and rich in cysteine-like 1 (SPARCL1) is down regulated in aggressive prostate cancers and is prognostic for poor clinical outcome. Proc Natl Acad Sci USA 109 14977-14982, 2012.

13. Bendik I, Schraml P and Ludwig CU: Characterization of MAST9/Hevin, a SPARC-like protein, that is down-regulated in non-small cell lung cancer. Cancer Res 58: 626-629, 1998.

14. Nelson PS, Plymate SR, Wang K, True LD, Ware JL, Gan L, Liu AY and Hood L: Hevin, an antiadhesive extracellular matrix protein, is down-regulated in metastatic prostate adenocarcinoma. Cancer Res 58: 232-236, 1998.

15. Zaravinos A, Lambrou GI, Boulalas I, Delakas D and Spandidos DA: Identification of common differentially expressed genes in urinary bladder cancer. PLoS One 6: e18135, 2011.

16. Esposito I, Kayed H, Keleg S, Giese T, Sage EH, Schirmacher P, Friess $H$ and Kleeff J: Tumor-suppressor function of SPARC-like protein $1 /$ Hevin in pancreatic cancer. Neoplasia 9: 8-17, 2007.

17. Xiang Y, Qiu Q, Jiang M, Jin R, Lehmann BD, Strand DW, Jovanovic B, DeGraff DJ, Zheng Y, Yousif DA, et al: SPARCL1 suppresses metastasis in prostate cancer. Mol Oncol 7: 1019-1030, 2013.

18. Hurley PJ, Hughes RM, Simons BW, et al: Androgen-Regulated SPARCL1 in the tumor microenvironment inhibits metastatic progression. Cancer Res 75: 4322-4334, 2015.

19. Sivaraman VS, Wang $H$, Nuovo GJ and Malbon CC: Hyperexpression of mitogen-activated protein kinase in human breast cancer. J Clin Invest 99: 1478-1483, 1997.

20. Hoshino R, Chatani Y, Yamori T, Tsuruo T, Oka H, Yoshida O, Shimada Y, Ari-i S, Wada H, Fujimoto J and Kohno M: Constitutive activation of the 41-/43-kDa mitogen-activated protein kinase signaling pathway in human tumors. Oncogene 18: 813-822, 1999

21. Eliceiri BP, Klemke R, Strömblad S and Cheresh DA: Integrin alphavbeta3 requirement for sustained mitogen-activated protein kinase activity during angiogenesis. J Cell Bio 140: 1255-1263, 1998.

22. Mandell JW, Hussaini IM, Zecevic M, Weber MJ and VandenBerg SR: In situ visualization of intratumor growth factor signaling: Immunohistochemical localization of activated ERK/MAP kinase in glial neoplasms. Am J Pathol 153: $1411-1423,1998$
23. Røe OD, Szulkin A, Anderssen E, Flatberg A, Sandeck H, Amundsen T, Erlandsen SE, Dobra K and Sundstrøm SH: Molecular resistance fingerprint of pemetrexed and platinum in a long-term survivor of mesothelioma. PLoS One 7: e40521, 2012.

24. Huang RL, Yuan Y, Tu J, Zou GM and Li Q Opposing TNF- $\alpha /$ IL- $1 \beta$ - and BMP-2-activated MAPK signaling pathways converge on Runx2 to regulate BMP-2-induced osteoblastic differentiation. Cell Death Dis 5: e1187, 2014

25. Guo C, Yang XG, Wang F and Ma XY: IL-1 $\alpha$ induces apoptosis and inhibits the osteoblast differentiation of MC3T3-E1 cells through the JNK and p38 MAPK pathways. Int J Mol Med 38 319-327, 2016.

26. Kyosseva SV: Targeting MAPK signaling in Age-related macular degeneration. Ophthalmol Eye Dis 8: 23-30, 2016.

27. Lim W, Jeong M, Bazer FW and Song G: Coumestrol inhibits proliferation and migration of prostate cancer cells by regulating AKT, ERK1/2, and JNK MAPK cell signaling cascades. J Cell Physiol 232: 862-871, 2017

28. Wang Y, Gao C, Zhang Y, Gao J, Teng F, Tian W, Yang W, Yan Y and Xue F: Visfatin stimulates endometrial cancer cell proliferation via activation of PI3K/Akt and MAPK/ERK1/2 signalling pathways. Gynecol Oncol 143: 168-178, 2016.

29. Yang A, Fan H, Zhao Y, Zha X, Zhang H, Hu Z and Tu P: Huaier aqueous extract inhibits proliferation and metastasis of tuberous sclerosis complex cell models through downregulation of JAK2/STAT3 and MAPK signaling pathways. Oncol Rep 36: 1491-1498, 2016

30. Yue X, Wu M, Jiang H, Hao J, Zhao Q, Zhu Q, Saren G, Zhang Y and Zhang $\mathrm{X}$ : Endothelial lipase is upregulated by interleukin-6 partly via the p38 MAPK and p65 NF-kB signaling pathways. Mol Med Rep 14: 1979-1985, 2016

31. Koyani CN, Kitz K, Rossmann C, Bernhart E, Huber E, Trummer C, Windischhofer W, Sattler W and Malle E: Activation of the MAPK/Akt/Nrf2-Egr1/HO-1-GCLc axis protects MG-63 osteosarcoma cells against 15d-PGJ2-mediated cell death. Biochem Pharmacol 104: 29-41, 2016.

32. Lien LM, Wang MJ, Chen RJ, Chiu HC, Wu JL, Shen MY, Chou DS, Sheu JR, Lin KH and Lu WJ: Nobiletin, a polymethoxylated flavone, inhibits glioma cell growth and migration via arresting cell cycle and suppressing MAPK and Akt pathways. Phytother Res 30: 214-221,2016.

33. Safia, Kamil M, Jadiya P, Sheikh S, Haque E, Nazir A, Lakshmi V and Mir SS: The chromone alkaloid, rohitukine, affords anti-cancer activity via modulating apoptosis pathways in A549 cell line and yeast mitogen activated protein kinase (MAPK) pathway. PLoS One 10: e0137991, 2015.

34. Atiq R, Hertz R, Eldad S, Smeir E and Bar-Tana J: Suppression of B-Raf(V600E) cancers by MAPK hyper-activation. Oncotarget 7: 18694-18704, 2016.

35. Chakraborty C, Sharma AR, Patra BC, Bhattacharya M, Sharma G and Lee SS: MicroRNAs mediated regulation of MAPK signaling pathways in chronic myeloid leukemia. Oncotarget 7: 42683-42697, 2016.

36. Li L, Wen XZ, Bu ZD, Cheng XJ, Xing XF, Wang XH, Zhang LH, Guo T, Du H, Hu Y, et al: Paclitaxel enhances tumoricidal potential of TRAIL via inhibition of MAPK in resistant gastric cancer cells. Oncol Rep 35: 3009-3017, 2016.

37. Li QC, Liang Y, Tian Y and Hu GR: Arctigenin induces apoptosis in colon cancer cells through ROS/p38MAPK pathway. J BUON 21: 87-94, 2016 .

38. Lin L and Bivona TG: The Hippo effector YAP regulates the response of cancer cells to MAPK pathway inhibitors. Mol Cell Oncol 3: e1021441, 2015.

39. Chang L and Karin M: Mammalian MAP kinase signalling cascades. Nature 410: 37-40, 2001

40. Johnson GL and Lapadat R: Mitogen-activated protein kinase pathways mediated by ERK, JNK, and p38 protein kinases. Science 298: 1911-1912, 2002.

41. Dunn KL, Espino PS, Drobic B, He S and Davie JR: The Ras-MAPK signal transduction pathway, cancer and chromatin remodeling. Biochem Cell Biol 83: 1-14, 2005.

42. Oka H, Chatani Y, Hoshino R, Ogawa O, Kakehi Y, Terachi T, Okada Y, Kawaichi M, Kohno M and Yoshida O: Constitutive activation of mitogen-activated protein (MAP) kinases in human renal cell carcinoma. Cancer Res 55: 4182-4187, 1995. 